\title{
Entire solutions for several second-order partial differential-difference equations of Fermat type with two complex variables
}

Hong Yan $\mathrm{Xu}^{1,2^{*}}$, Da Wei Meng ${ }^{2}$, Sanyang Liu ${ }^{2}$ and Hua Wang ${ }^{3}$

"Correspondence:

xuhongyanxidian@126.com

1 School of Mathematics and

Computer Science, Shangrao

Normal University, Shangrao, Jiangxi

334001, P.R. China

${ }^{2}$ School of Mathematics and

Statistics, Xidian University, Xi'an,

Shaanxi 710126, P.R. China

Full list of author information is

available at the end of the article

\section{Springer}

\begin{abstract}
This paper is concerned with description of the existence and the forms of entire solutions of several second-order partial differential-difference equations with more general forms of Fermat type. By utilizing the Nevanlinna theory of meromorphic functions in several complex variables we obtain some results on the forms of entire solutions for these equations, which are some extensions and generalizations of the previous theorems given by Xu and Cao (Mediterr. J. Math. 15:1-14, 2018; Mediterr. J. Math. 17:1-4, 2020) and Liu et al. (J. Math. Anal. Appl. 359:384-393, 2009; Electron. J. Differ. Equ. 2013:59-110, 2013; Arch. Math. 99:147-155, 2012). Moreover, by some examples we show the existence of transcendental entire solutions with finite order of such equations.
\end{abstract}

MSC: 30D35; 35M30; 32W50; 39A45

Keywords: Nevanlinna theory; Existence; Entire solution; Partial differential-difference equation

\section{Introduction}

The main purpose of this paper is investigation of the existence and the forms of transcendental entire solutions with finite order of second-order differential difference equations

$$
\left(\frac{\partial^{2} f\left(z_{1}, z_{2}\right)}{\partial z_{1}^{2}}\right)^{2}+f\left(z_{1}+c_{1}, z_{2}+c_{2}\right)^{2}=e^{g\left(z_{1}, z_{2}\right)}
$$

and

$$
\left(\frac{\partial^{2} f\left(z_{1}, z_{2}\right)}{\partial z_{1}^{2}}\right)^{2}+\left[f\left(z_{1}+c_{1}, z_{2}+c_{2}\right)-f\left(z_{1}, z_{2}\right)\right]^{2}=e^{g\left(z_{1}, z_{2}\right)}
$$

where $g\left(z_{1}, z_{2}\right)$ is a polynomial in $\mathbb{C}^{2}$. In general, for the Fermat-type functional equation

$$
f^{m}+g^{n}=1
$$

(c) The Author(s) 2021. This article is licensed under a Creative Commons Attribution 4.0 International License, which permits use, sharing, adaptation, distribution and reproduction in any medium or format, as long as you give appropriate credit to the original author(s) and the source, provide a link to the Creative Commons licence, and indicate if changes were made. The images or other third party material in this article are included in the article's Creative Commons licence, unless indicated otherwise in a credit line to the material. If material is not included in the article's Creative Commons licence and your intended use is not permitted by statutory regulation or exceeds the permitted use, you will need to obtain permission directly from the copyright holder. To view a copy of this licence, visit http://creativecommons.org/licenses/by/4.0/. 
Gross [6] discussed the existence of solutions of equation (1.1) and showed that the entire solutions are $f=\cos a(z), g=\sin a(z)$ for $m=n=2$, where $a(z)$ is an entire function. Montel [7] proved that there are no nonconstant entire solutions for equation (1.1) for $m=n>2$.

Recently, Han and Lü [8] gave a description of meromorphic solutions for the functional equation (1.1) when $g(z)=f^{\prime}(z), m=n$, and 1 is replaced by $e^{\alpha z+\beta}$, where $\alpha, \beta \in \mathbb{C}$, and obtained the following results.

Thereom A (See [8, Theorem 1.1]) The meromorphic solutions $f$ of the differential equation

$$
f^{n}(z)+\left(f^{\prime}\right)^{n}(z)=e^{\alpha z+\beta}
$$

must be entire functions, and the following statements hold:

(A) For $n=1$, the general solutions of (1.2) are $f(z)=\frac{e^{\alpha z+\beta}}{\alpha+1}+a e^{-z}$ for $\alpha \neq-1$ and

$$
f(z)=z e^{-z+\beta}+a e^{-z} \text {. }
$$

(B) For $n=2$, either $\alpha=0$ and the general solutions of (1.2) are $f(z)=e^{\frac{\beta}{2}} \sin (z+b)$, or

$$
f(z)=d e^{\frac{\alpha z+\beta}{2}} \text {. }
$$

(C) For $n \geq 3$, the general solutions of (1.2) are $f(z)=d e^{\frac{\alpha z+\beta}{n}}$.

Here $\alpha, \beta, a, b, d \in \mathbb{C}$ with $d^{n}\left(1+\left(\frac{\alpha}{n}\right)^{n}\right)=1$ for $n \geq 1$.

They also proved that all the trivial meromorphic solutions of $f^{n}(z)+f^{n}(z+c)=e^{\alpha z+\beta}$ are the functions $f(z)=d e^{\frac{\alpha z+\beta}{n}}$ with $d^{n}\left(1+e^{\alpha c}\right)=1$ for $n \geq 1$ (see [8, p. 99]).

An equation is called differential-difference equation (DDE) if the equation includes derivatives and shifts or differences of $f$ (see [9]). In many previous papers [10-15], Naftalevich [11, 12] in 1995 discussed the meromorphic solutions of complex differentialdifference equations with one complex variable by using the operator theory and iteration method, but recently, many researchers have begun to discuss this kind of equations by using the difference analogues of Nevanlinna theory (see [16-19]). In particular, Liu et al. [3-5] investigated the existence of entire solutions with finite order of the Fermat-type differential-difference equations

$$
\begin{aligned}
& f^{\prime}(z)^{2}+f(z+c)^{2}=1, \\
& f^{\prime}(z)^{2}+[f(z+c)-f(z)]^{2}=1 .
\end{aligned}
$$

They proved that the transcendental entire solutions with finite order of equation (1.3) must satisfy $f(z)=\sin (z \pm B i)$, where $B$ is a constant, $c=2 k \pi$ or $c=(2 k+1) \pi$ with integer $k$, and the transcendental entire solutions with finite order of equation (1.4) must satisfy $f(z)=12 \sin (2 z+B i)$, where $c=(2 k+1) \pi$ with integer $k$, and $B$ is a constant. In 2019, Liu and Gao [20] further studied the entire solutions of second-order differential and difference equation with single complex variable and obtained the following:

Thereom B (See [20, Theorem 2.1]) Let $f$ be a transcendental entire solution with finite order of the complex differential-difference equation

$$
f^{\prime \prime}(z)^{2}+f(z+c)^{2}=Q(z)
$$


Then $Q(z)=c_{1} c_{2}$ is a constant, and $f(z)$ satisfies

$$
f(z)=\frac{c_{1} e^{a z+b}+c_{2} e^{-a z-b}}{2 a^{2}},
$$

with $a, b \in \mathbb{C}$ such that $a^{4}=1$ and $c=\frac{\log \left(-i a^{2}\right)+2 k \pi i}{a}, k \in \mathbb{Z}$.

Now let us recall some previous results on Fermat-type partial differential equations with several complex variables (including [21-25]). Khavinson [22] in 1995 pointed out that any entire solution of the partial differential equation $\left(\frac{\partial f}{\partial z_{1}}\right)^{2}+\left(\frac{\partial f}{\partial z_{2}}\right)^{2}=1$ in $\mathbb{C}^{2}$ is necessarily linear. This partial differential equation in real variable case occurs in the study of characteristic surfaces and wave propagation theory, and it is the two-dimensional eiconal equation, one of the main equations of geometric optics (see [26, 27]). In 2005, Li [28] discussed the partial differential equation of Fermat-type

$$
\left(\frac{\partial u}{\partial z_{1}}\right)^{2}+\left(\frac{\partial u}{\partial z_{2}}\right)^{2}=e^{g}
$$

where $g$ is a polynomial or an entire function in $\mathbb{C}^{2}$, and obtained some results on the forms of entire solution of equation (1.5).

Thereom C ([28, Theorem 2.1]) Let $g$ be a polynomial in $\mathbb{C}^{2}$. Then $u$ is an entire solution of the partial differential equation (1.5) if and only if

(i) $u=f\left(c_{1} z_{1}+c_{2} z_{2}\right)$; or

(ii) $u=\phi_{1}\left(z_{1}+i z_{2}\right)+\phi_{2}\left(z_{1}-i z_{2}\right)$,

where $f$ is an entire function in $\mathbb{C}$ satisfying $f^{\prime}\left(c_{1} z_{1}+c_{2} z_{2}\right)= \pm e^{\frac{1}{2} g(z)}, c_{1}$ and $c_{2}$ are two constants satisfying $c_{1}^{2}+c_{2}^{2}=1$, and $\phi_{1}$ and $\phi_{2}$ are entire functions in $\mathbb{C}$ satisfying $\phi_{1}^{\prime}\left(z_{1}+\right.$ $\left.i z_{2}\right) \phi_{2}^{\prime}\left(z_{1}-i z_{2}\right)=\frac{1}{4} e^{g(z)}$.

Very recently, Xu and Cao [1, 2, 29] investigated the existence of solutions for some Fermat-type partial differential-difference equations with several variables by using the difference logarithmic derivative lemma of several complex variables and obtained the following theorem (see [29-31]).

Thereom $\mathbf{D}$ (See [1, Theorem 1.2]) Let $c=\left(c_{1}, c_{2}\right)$ be a constant in $\mathbb{C}^{2}$. Then any transcendental entire solution with finite order of the partial differential-difference equation

$$
\left(\frac{\partial f\left(z_{1}, z_{2}\right)}{\partial z_{1}}\right)^{2}+f\left(z_{1}+c_{1}, z_{2}+c_{2}\right)^{2}=1
$$

has the form of $f\left(z_{1}, z_{2}\right)=\sin \left(A z_{1}+B\right)$, where $A \in \mathbb{C}$ is a constant satisfying $A e^{i A c_{1}}=1$, and $B \in \mathbb{C}$ is a constant; in the particular case $c_{1}=0$, we have $f\left(z_{1}, z_{2}\right)=\sin \left(z_{1}+B\right)$.

Theorems B, C, and D suggest the following questions as open problems.

Question 1.1 What will happen when the right side of those equations, 1, is replaced by a function $e^{g}$ in Theorem $\mathrm{D}$, where $g$ is a polynomial in $\mathbb{C}^{2}$ ?

Question 1.2 What will happen when $\frac{\partial f\left(z_{1}, z_{2}\right)}{\partial z_{1}}$ is replaced by $\frac{\partial^{2} f\left(z_{1}, z_{2}\right)}{\partial z_{1}^{2}}$ or $\frac{\partial^{2} f\left(z_{1}, z_{2}\right)}{\partial z_{1} \partial z_{2}}$ in Theorem $\mathrm{D}$ ? 


\section{Results and some examples}

In view of the above questions, this paper is concerned with description of entire solutions for several second-order partial differential-difference equations of Fermat type of more general form. The main tools used in this paper are the Nevanlinna theory and difference Nevanlinna theory with several complex variables. Our principal results generalize the previous theorems given by $\mathrm{Xu}$ and Cao [1] and Liu, Cao, and Cao [5]. Throughout this paper, for convenience, we assume that $z+w=\left(z_{1}+w_{1}, z_{2}+w_{2}\right)$ for any $z=\left(z_{1}, z_{2}\right), w=$ $\left(w_{1}, w_{2}\right)$. We now state the main results of this paper.

Theorem 2.1 Let $c=\left(c_{1}, c_{2}\right) \in \mathbb{C}^{2}$ and $c_{2} \neq 0$. If the partial differential-difference equation

$$
\left(\frac{\partial^{2} f\left(z_{1}, z_{2}\right)}{\partial z_{1}^{2}}\right)^{2}+f\left(z_{1}+c_{1}, z_{2}+c_{2}\right)^{2}=e^{g\left(z_{1}, z_{2}\right)}
$$

admits a transcendental entire solution $f\left(z_{1}, z_{2}\right)$ of finite order, then $g\left(z_{1}, z_{2}\right)$ must be a linear function of the form $g\left(z_{1}, z_{2}\right)=A_{1} z_{1}+A_{2} z_{2}+B$, where $A_{1}, A_{2}, B \in \mathbb{C}$. Further, $f\left(z_{1}, z_{2}\right)$ must satisfy one of the following cases:

(i)

$$
f\left(z_{1}, z_{2}\right)=\frac{4\left(\xi^{2}+1\right)}{A_{1}^{2} \xi} e^{\frac{1}{2} g\left(z_{1}, z_{2}\right)},
$$

with $\xi(\neq 0), A_{1}, A_{2}, B \in \mathbb{C}$ satisfying

$$
\frac{\xi^{2}-1}{4\left(\xi^{2}+1\right) i} A_{1}^{2}=e^{\frac{1}{2}\left(A_{1} c_{1}+A_{2} c_{2}\right)}
$$

(ii)

$$
f\left(z_{1}, z_{2}\right)=\frac{A_{21}^{2} e^{L_{1}(z)+B_{1}}+A_{11}^{2} e^{L_{2}(z)+B_{2}}}{2 A_{11}^{2} A_{21}^{2}}
$$

where $L_{1}(z)=A_{11} z_{1}+A_{12} z_{2}+B_{1}, L_{2}(z)=A_{21} z_{1}+A_{22} z_{2}+B_{2}, A_{j 1}, A_{j 2}, B_{j} \in \mathbb{C}(j=1,2)$ satisfy

$$
L_{1}(z) \neq L_{2}(z), \quad g(z)=L_{1}(z)+L_{2}(z)+B_{1}+B_{2},
$$

and

$$
-i A_{11}^{2} e^{-L_{1}(c)}=i A_{21}^{2} e^{-L_{2}(c)}=1 .
$$

The following examples show that the forms of solutions are precise to some extent.

Example 2.1 Let $A_{1}=2, A_{2}=1, B=0$, and

$$
f\left(z_{1}, z_{2}\right)=\frac{\sqrt{2}}{2} e^{z_{1}+\frac{1}{2} z_{2}} .
$$

Then $\rho(f)=1$, and $f\left(z_{1}, z_{2}\right)$ is a transcendental entire solution of equation (2.1) with $g(z)=$ $2 z_{1}+z_{2}, c_{1}=\pi i$, and $c_{2}=2 \pi i$. 
Example 2.2 Let $L_{1}(z)=i z_{1}+\frac{1}{2} z_{2}, L_{2}(z)=-i z_{1}-\frac{5}{2} z_{2}, B_{1}=B_{2}=0$, and

$$
f\left(z_{1}, z_{2}\right)=-\frac{e^{i z_{1}+\frac{1}{2} z_{2}}+e^{-i z_{1}-\frac{5}{2} z_{2}}}{2}
$$

Then $\rho(f)=1$, and $f\left(z_{1}, z_{2}\right)$ is a transcendental entire solution of equation (2.1) with $g(z)=$ $-\frac{3}{2} z_{2}, c_{1}=\pi$, and $c_{2}=-\pi i$.

From Theorem 2.1 we easily get the following:

Corollary 2.1 Let $c=\left(c_{1}, c_{2}\right) \in \mathbb{C}^{2}, c_{2} \neq 0$, and let $g\left(z_{1}, z_{2}\right)$ be not a linear function of the form $L(z)=A_{1} z_{1}+A_{2} z_{2}+B$, where $A_{1}, A_{2}, B \in \mathbb{C}$. Then the partial differential-difference equation

$$
\left(\frac{\partial^{2} f\left(z_{1}, z_{2}\right)}{\partial z_{1}^{2}}\right)^{2}+f\left(z_{1}+c_{1}, z_{2}+c_{2}\right)^{2}=e^{g\left(z_{1}, z_{2}\right)}
$$

admits no transcendental entire solution of finite order.

The following example shows that the condition $c_{2} \neq 0$ in Corollary 2.1 cannot be removed.

Example 2.3 Let $f\left(z_{1}, z_{2}\right)=\frac{\sqrt{2}}{2} e^{z_{1}+z_{2}-2 \pi z_{2}^{2}}$. Then $f\left(z_{1}, z_{2}\right)$ is a transcendental entire solution of finite order of equation (2.2) with $c=\left(c_{1}, c_{2}\right)=(2 \pi i, 0)$ and $g\left(z_{1}, z_{2}\right)=2 z_{1}+2 z_{2}-4 \pi z_{2}^{2}$.

Remark 2.1 In addition, in view of Theorem 2.1, we can obtain the conclusions of Theorem 1.2 in [1] if $\alpha=1, \beta=0$, and $g(z)=2 k \pi i, k \in \mathbb{Z}$, in equation (2.1).

For the difference counterpart of Theorem 2.1, we have the following:

Theorem 2.2 Let $c=\left(c_{1}, c_{2}\right) \in \mathbb{C}^{2}, c_{2} \neq 0$. If the partial differential-difference equation

$$
\left(\frac{\partial^{2} f\left(z_{1}, z_{2}\right)}{\partial z_{1}^{2}}\right)^{2}+\left[f\left(z_{1}+c_{1}, z_{2}+c_{2}\right)-f\left(z_{1}, z_{2}\right)\right]^{2}=e^{g\left(z_{1}, z_{2}\right)}
$$

admits a transcendental entire solution $f\left(z_{1}, z_{2}\right)$ of finite order, then $g\left(z_{1}, z_{2}\right)$ must be a linear function of the form $g\left(z_{1}, z_{2}\right)=A_{1} z_{1}+A_{2} z_{2}+B$ with $A_{1}, A_{2}, B \in \mathbb{C}$. Further, $f\left(z_{1}, z_{2}\right)$ must satisfy one of the following cases:

(i)

$$
f\left(z_{1}, z_{2}\right)=\frac{4\left(\xi^{2}+1\right)}{A_{1}^{2} \xi} e^{\frac{1}{2} g\left(z_{1}, z_{2}\right)}+z_{1} G_{1}\left(z_{2}\right)+G_{2}\left(z_{2}\right)
$$

where $G_{1}\left(z_{2}\right)$ is a finite-order entire period function in $z_{2}$ with period $c_{2}, \xi(\neq 0)$, $A_{1}, A_{2}, B \in \mathbb{C}$ satisfying

$$
G_{2}\left(z_{2}+c_{2}\right)=G_{2}\left(z_{2}\right)-c_{1} G_{1}\left(z_{2}\right), \quad \frac{\xi^{2}-1}{2 i\left(\xi^{2}+1\right)} A_{1}^{2}+1=e^{\frac{1}{2}\left(A_{1} c_{1}+A_{2} c_{2}\right)}=e^{\frac{1}{2} g\left(c_{1}, c_{2}\right)}
$$


(ii)

$$
f\left(z_{1}, z_{2}\right)=\frac{A_{21}^{2} e^{L_{1}(z)+B_{1}}+A_{11}^{2} e^{L_{2}(z)+B_{2}}}{2 A_{11}^{2} A_{21}^{2}}+z_{1} G_{1}\left(z_{2}\right)+G_{2}\left(z_{2}\right),
$$

where $G_{1}\left(z_{2}\right)$ is a finite-order entire period function in $z_{2}$ with period $c_{2}$, $L_{1}(z)=A_{11} z_{1}+A_{12} z_{2}+B_{1}, L_{2}(z)=A_{21} z_{1}+A_{22} z_{2}+B_{2}, A_{i j}, B_{i} \in \mathbb{C}$ satisfy

$$
\begin{aligned}
& G_{2}\left(z_{2}+c_{2}\right)=G_{2}\left(z_{2}\right)-c_{1} G_{1}\left(z_{2}\right), \quad L_{1}(z) \neq L_{2}(z), \\
& g(z)=L_{1}(z)+L_{2}(z)+B_{1}+B_{2},
\end{aligned}
$$

and

$$
\left(1-i A_{11}^{2}\right) e^{-\left(A_{11} c_{1}+A_{12} c_{2}\right)}=1, \quad\left(1+i A_{21}^{2}\right) e^{-\left(A_{21} c_{1}+A_{22} c_{2}\right)}=1 .
$$

The following examples explain the existence of transcendental finite-order entire solutions of (2.3).

Example 2.4 Let $A_{1}=2, A_{2}=-1, G_{1}\left(z_{2}\right)=e^{z_{2}}, G_{2}\left(z_{2}\right)=e^{2 z_{2}}-z_{2} e^{z_{2}}, B=0$, and

$$
f\left(z_{1}, z_{2}\right)=\frac{\sqrt{5}}{5} e^{z_{1}-\frac{1}{2} z_{2}}+\left(z_{1}-z_{2}\right) e^{z_{2}}+e^{2 z_{2}} .
$$

Then $\rho(f)=1$, and $f\left(z_{1}, z_{2}\right)$ is a transcendental entire solution of equation (2.3) with $g(z)=$ $2 z_{1}-z_{2}, c_{1}=2 \pi i$, and $c_{2}=2 \pi i$.

Example 2.5 Let $L_{1}(z)=z_{1}+z_{2}, L_{2}(z)=z_{1}-z_{2}, G_{1}(z)=e^{\frac{4 \pi i}{\log (-i)} z_{2}}, G_{2}\left(z_{2}\right)=-\frac{\log (-i)}{\log 2} e^{\frac{4 \pi i z_{2}}{\log (-i)}}$, $B_{1}=B_{2}=0$, and

$$
f\left(z_{1}, z_{2}\right)=\frac{e^{z_{1}+z_{2}}+e^{z_{1}-z_{2}}}{2}+\left(z_{1}-\frac{\log (-i)}{\log 2} z_{2}\right) e^{\frac{4 \pi i}{\log (-i)} z_{2}}
$$

Then $f\left(z_{1}, z_{2}\right)$ is a transcendental finite-order entire solution of equation (2.3) with $g(z)=$ $2 z_{1}, c_{1}=\frac{1}{2} \log 2$, and $c_{2}=\frac{1}{2} \log (-i)$.

In view of Theorem 2.2, we obtain the following:

Corollary 2.2 Let $c=\left(c_{1}, c_{2}\right) \in \mathbb{C}^{2}, c_{2} \neq 0$, and let $g\left(z_{1}, z_{2}\right)$ be not a linear function of the form $L(z)=A_{1} z_{1}+A_{2} z_{2}+B$ with $A_{1}, A_{2}, B \in \mathbb{C}$. Then the partial differential-difference equation

$$
\left(\frac{\partial^{2} f\left(z_{1}, z_{2}\right)}{\partial z_{1}^{2}}\right)^{2}+\left[f\left(z_{1}+c_{1}, z_{2}+c_{2}\right)-f\left(z_{1}, z_{2}\right)\right]^{2}=e^{g\left(z_{1}, z_{2}\right)}
$$

has no transcendental entire solution of finite order.

The following example shows that the condition $c_{2} \neq 0$ in Corollary 2.2 cannot be removed. 
Example 2.6 Let $f\left(z_{1}, z_{2}\right)=e^{z_{1}+z_{2}-4 \pi i z_{2}^{3}}$. Then $f\left(z_{1}, z_{2}\right)$ is a transcendental finite-order entire solution of equation (2.4) with $c=\left(c_{1}, c_{2}\right)=(2 \pi i, 0)$ and $g\left(z_{1}, z_{2}\right)=2 z_{1}+2 z_{2}-8 \pi i z_{2}^{3}$.

When $\frac{\partial^{2} f\left(z_{1}, z_{2}\right)}{\partial z_{1}^{2}}$ is replaced by $\frac{\partial^{2} f\left(z_{1}, z_{2}\right)}{\partial z_{1} \partial z_{2}}$ in Theorems 2.1 and 2.2, we have the following:

Theorem 2.3 Let $c=\left(c_{1}, c_{2}\right) \in \mathbb{C}^{2}, c_{1} \neq 0, c_{2} \neq 0$. If the partial differential-difference equation

$$
\left(\frac{\partial^{2} f\left(z_{1}, z_{2}\right)}{\partial z_{1} \partial z_{2}}\right)^{2}+f\left(z_{1}+c_{1}, z_{2}+c_{2}\right)^{2}=e^{g\left(z_{1}, z_{2}\right)}
$$

admits a transcendental entire solution of finite order, then $g\left(z_{1}, z_{2}\right)$ must be a linear function of the form $g\left(z_{1}, z_{2}\right)=A_{1} z_{1}+A_{2} z_{2}+B$ with $A_{1}, A_{2}, B \in \mathbb{C}$. Further, $f\left(z_{1}, z_{2}\right)$ must satisfy one of the following cases:

(i)

$$
f\left(z_{1}, z_{2}\right)=\frac{4\left(\xi^{2}+1\right)}{A_{1} A_{2} \xi} e^{\frac{1}{2} g\left(z_{1}, z_{2}\right)}
$$

with $\xi(\neq 0), A_{1}, A_{2}, B \in \mathbb{C}$ satisfying

$$
\frac{\xi^{2}-1}{4\left(\xi^{2}+1\right) i} A_{1} A_{2}=e^{\frac{1}{2}\left(A_{1} c_{1}+A_{2} c_{2}\right)}
$$

(ii)

$$
f\left(z_{1}, z_{2}\right)=\frac{A_{21} A_{22} e^{L_{1}(z)+B_{1}}+A_{11} A_{12} e^{L_{2}(z)+B_{2}}}{2 A_{11} A_{12} A_{21} A_{22}},
$$

where $L_{1}(z)=A_{11} z_{1}+A_{12} z_{2}+B_{1}, L_{2}(z)=A_{21} z_{1}+A_{22} z_{2}+B_{2}, A_{j 1}, A_{j 2}, B_{j} \in \mathbb{C}(j=1,2)$ satisfy

$$
L_{1}(z) \neq L_{2}(z), \quad g(z)=L_{1}(z)+L_{2}(z)+B_{1}+B_{2},
$$

and

$$
-i A_{11} A_{12} e^{-L_{1}(c)}=i A_{21} A_{22} e^{-L_{2}(c)}=1 .
$$

Example 2.7 Let $A_{1}=2, A_{2}=2, B=0$, and

$$
f\left(z_{1}, z_{2}\right)=\frac{\sqrt{2}}{2} e^{z_{1}+z_{2}} .
$$

Then $\rho(f)=1$, and $f\left(z_{1}, z_{2}\right)$ is a transcendental entire solution of equation (2.5) with $g(z)=$ $2 z_{1}+2 z_{2}, c_{1}=\pi i$, and $c_{2}=\pi i$.

Example 2.8 Let $L_{1}(z)=z_{1}+z_{2}, L_{2}(z)=z_{1}-z_{2}, B_{1}=B_{2}=0$, and

$$
f\left(z_{1}, z_{2}\right)=\frac{e^{z_{1}+z_{2}}-e^{z_{1}-z_{2}}}{2} .
$$


Then $\rho(f)=1$, and $f\left(z_{1}, z_{2}\right)$ is a transcendental entire solution of equation (2.5) with $g(z)=$ $2 z_{1}, c_{1}=\frac{\pi}{2} i$, and $c_{2}=\pi i$.

From Theorem 2.3 we get the following:

Corollary 2.3 Let $c=\left(c_{1}, c_{2}\right) \in \mathbb{C}^{2}, c_{1} \neq 0, c_{2} \neq 0$, and let $g\left(z_{1}, z_{2}\right)$ be not a linear function of the form $L(z)=A_{1} z_{1}+A_{2} z_{2}+B$ with $A_{1}, A_{2}, B \in \mathbb{C}$. Then the partial differential-difference equation

$$
\left(\frac{\partial^{2} f\left(z_{1}, z_{2}\right)}{\partial z_{1} \partial z_{2}}\right)^{2}+f\left(z_{1}+c_{1}, z_{2}+c_{2}\right)^{2}=e^{g\left(z_{1}, z_{2}\right)}
$$

admits no transcendental entire solution of finite order.

The following example shows that the condition $c_{1} \neq 0, c_{2} \neq 0$ in Corollary 2.3 cannot be removed.

Example 2.9 Let $f\left(z_{1}, z_{2}\right)=e^{z_{2}+z_{2}^{3}}$. Then $f\left(z_{1}, z_{2}\right)$ is a transcendental finite-order entire solution of equation (2.6) with $c=\left(c_{1}, c_{2}\right)=(2 \pi i, 0)$ and $g\left(z_{1}, z_{2}\right)=2 z_{2}+2 z_{2}^{3}$.

Theorem 2.4 Let $c=\left(c_{1}, c_{2}\right) \neq(0,0) \in \mathbb{C}^{2}$. If the partial differential-difference equation

$$
\left(\frac{\partial^{2} f\left(z_{1}, z_{2}\right)}{\partial z_{1} \partial z_{2}}\right)^{2}+\left[f\left(z_{1}+c_{1}, z_{2}+c_{2}\right)-f\left(z_{1}, z_{2}\right)\right]^{2}=e^{g\left(z_{1}, z_{2}\right)}
$$

admits a transcendental entire solution of finite order, then $g\left(z_{1}, z_{2}\right)$ must be a linear function of the form $g\left(z_{1}, z_{2}\right)=A_{1} z_{1}+A_{2} z_{2}+B$ with $A_{1}, A_{2}, B \in \mathbb{C}$. Further, $f\left(z_{1}, z_{2}\right)$ must satisfy one of the following cases:

(i)

$$
f\left(z_{1}, z_{2}\right)=\frac{4\left(\xi^{2}+1\right)}{A_{1} A_{2} \xi} e^{\frac{1}{2} g\left(z_{1}, z_{2}\right)}+G_{3}\left(z_{1}\right)+D_{1} z_{1}+G_{4}\left(z_{2}\right)+D_{2} z_{2}
$$

where $G_{3}\left(z_{1}\right)$ and $G_{4}\left(z_{2}\right)$ are finite-order entire periodic functions in $z_{1}$ and $z_{2}$ with periods $c_{1}$ and $c_{2}$, respectively, and $\xi(\neq 0), A_{1}, A_{2}, B, D_{1}, D_{2} \in \mathbb{C}$ satisfy

$$
\frac{\xi^{2}-1}{4 i\left(\xi^{2}+1\right)} A_{1} A_{2}+1=e^{\frac{1}{2}\left(A_{1} c_{1}+A_{2} c_{2}\right)}=e^{\frac{1}{2} g\left(c_{1}, c_{2}\right)}, \quad D_{1} c_{1}+D_{2} c_{2}=0
$$

(ii)

$$
f\left(z_{1}, z_{2}\right)=\frac{A_{21} A_{22} e^{L_{1}(z)+B_{1}}+A_{11} A_{12} e^{L_{2}(z)+B_{2}}}{2 A_{11} A_{12} A_{21} A_{22}}+G_{3}\left(z_{1}\right)+D_{1} z_{1}+G_{4}\left(z_{2}\right)+D_{2} z_{2}
$$

where $G_{3}\left(z_{1}\right)$ and $G_{4}\left(z_{2}\right)$ are finite-order entire periodic functions in $z_{1}$ and $z_{2}$ with periods $c_{1}$ and $c_{2}$, respectively, $L_{1}(z)=A_{11} z_{1}+A_{12} z_{2}+B_{1}, L_{2}(z)=A_{21} z_{1}+A_{22} z_{2}+B_{2}$, $A_{j 1}, A_{j 2}, B_{j} \in \mathbb{C}(j=1,2)$ satisfy

$$
L_{1}(z) \neq L_{2}(z), \quad g(z)=L_{1}(z)+L_{2}(z)+B_{1}+B_{2}, \quad D_{1} c_{1}+D_{2} c_{2}=0,
$$


and

$$
\left(1-i A_{11} A_{12}\right) e^{-\left(A_{11} c_{1}+A_{12} c_{2}\right)}=1, \quad\left(1+i A_{21} A_{22}\right) e^{-\left(A_{21} c_{1}+A_{22} c_{2}\right)}=1 .
$$

Example 2.10 Let $A_{1}=2, A_{2}=2, B=0, G_{3}\left(z_{1}\right)=e^{2 z_{1}}, G_{4}\left(z_{2}\right)=e^{4 z_{2}}$, and

$$
f\left(z_{1}, z_{2}\right)=\frac{\sqrt{5}}{5} e^{2 z_{1}+2 z_{2}}+e^{2 z_{1}}+z_{1}+e^{4 z_{2}}+2 z_{2}
$$

Then $\rho(f)=1$, and $f\left(z_{1}, z_{2}\right)$ is a transcendental entire solution of equation (2.7) with $g(z)=$ $2 z_{1}+2 z_{2}, c_{1}=\pi i$, and $c_{2}=-\frac{\pi}{2} i$.

Example 2.11 Let $L_{1}(z)=z_{1}+z_{2}, L_{2}(z)=z_{1}-2 z_{2}, B_{1}=B_{2}=0, G_{3}(z)=e^{\frac{6 \pi i}{\log [-2(2+i)]} z_{1}}, G_{4}(z)=$ $e^{\frac{6 \pi i}{\log (1-i)-\log (1-2 i)} z_{2}}$, and

$$
f\left(z_{1}, z_{2}\right)=\frac{e^{z_{1}+z_{2}}}{2}-\frac{e^{z_{1}-2 z_{2}}}{4}+e^{\frac{6 \pi i}{\log [-2(2+i)]} z_{1}}-\frac{\log \frac{1-i}{1-2 i}}{\log (-2-2 i)} z_{1}+e^{\frac{6 \pi i}{\log (1-i)-\log (1-2 i)} z_{2}}
$$

Then $\rho(f)=1$, and $f\left(z_{1}, z_{2}\right)$ is a transcendental entire solution of equation (2.7) with $g(z)=$ $2 z_{1}-z_{2}, c_{1}=\frac{\log [-2(2+i)]}{3}$, and $c_{2}=\frac{\log (1-i)-\log (1-2 i)}{3}$.

In view of Theorem 2.4, we obtain the following:

Corollary 2.4 Let $c=\left(c_{1}, c_{2}\right) \neq(0,0) \in \mathbb{C}^{2}$, and let $g\left(z_{1}, z_{2}\right)$ be not a linear function of the form $L(z)=A_{1} z_{1}+A_{2} z_{2}+B$ with $A_{1}, A_{2}, B \in \mathbb{C}$. Then the partial differential-difference equation

$$
\left(\frac{\partial^{2} f\left(z_{1}, z_{2}\right)}{\partial z_{1} \partial z_{2}}\right)^{2}+\left[f\left(z_{1}+c_{1}, z_{2}+c_{2}\right)-f\left(z_{1}, z_{2}\right)\right]^{2}=e^{g\left(z_{1}, z_{2}\right)}
$$

admits no transcendental entire solution of finite order.

In view of Theorems 2.1 and 2.3, we also get the following:

Corollary 2.5 Letf be a finite-order transcendental entire solution of the partial differential equation

$$
\left(\frac{\partial^{2} f\left(z_{1}, z_{2}\right)}{\partial z_{1}^{2}}\right)^{2}+f\left(z_{1}, z_{2}\right)^{2}=1, \quad\left(\frac{\partial^{2} f\left(z_{1}, z_{2}\right)}{\partial z_{1} \partial z_{2}}\right)^{2}+f\left(z_{1}, z_{2}\right)^{2}=1
$$

Then $f\left(z_{1}, z_{2}\right)$ must be of the form

$$
f\left(z_{1}, z_{2}\right)=\frac{e^{L(z)+B}-e^{-L(z)-B}}{2 i}=\sin (-i(L(z)+B)),
$$

where $L(z)=A_{1} z_{1}+A_{2} z_{2}$ with $A_{1}, A_{2}, B \in \mathbb{C}$ satisfying $A_{1}^{4}=1$ and $A_{1}^{2} A_{2}^{2}=1$. 


\section{Some lemmas}

The following lemmas play the key role in proving our results.

Lemma 3.1 ([32, 33]) For an entire function $F$ on $\mathbb{C}^{n}$ with $F(0) \neq 0$, put $\rho\left(n_{F}\right)=\rho<\infty$. Then there exist a canonical function $f_{F}$ and a function $g_{F} \in \mathbb{C}^{n}$ such that $F(z)=f_{F}(z) e^{g_{F}(z)}$. For the particular case $n=1, f_{F}$ is the canonical Weierstrass product.

Remark 3.1 Here $\rho\left(n_{F}\right)$ is the order of the counting function of zeros of $F$.

Lemma 3.2 ([34]) If $g$ and $h$ are entire functions on the complex plane $\mathbb{C}$ and $g(h)$ is an entire function of finite order, then there are only two possible cases:

(a) the internal function $h$ is a polynomial, and the external function $g$ is of finite order;

(b) the internal function $h$ is not a polynomial but a function of finite order, and the external function $g$ is of zero order.

Lemma 3.3 ([35, Theorem 1.106]) Suppose that $a_{0}(z), a_{1}(z), \ldots, a_{n}(z)(n \geq 1)$ are meromorphic functions on $\mathbb{C}^{m}$ and $g_{0}(z), g_{1}(z), \ldots, g_{n}(z)$ are entire functions on $\mathbb{C}^{m}$ such that $g_{j}(z)-g_{k}(z)$ are not constants for $0 \leq j<k \leq n$. If

$$
\sum_{j=0}^{n} a_{j}(z) e^{g_{j}(z)} \equiv 0
$$

and

$$
\| T\left(r, a_{j}\right)=o(T(r)), \quad j=0,1, \ldots, n
$$

where $T(r)=\min _{0 \leq j<k \leq n} T\left(r, e^{g_{j}-g_{k}}\right)$, then $a_{j}(z) \equiv 0(j=0,1,2, \ldots, n)$.

Lemma 3.4 ([35, Lemma 3.1]) Let $f_{j}(\not \equiv 0), j=1,2,3$, be meromorphic functions on $\mathbb{C}^{m}$ such that $f_{1}$ is not constant, $f_{1}+f_{2}+f_{3}=1$, and

$$
\sum_{j=1}^{3}\left\{N_{2}\left(r, \frac{1}{f_{j}}\right)+2 \bar{N}\left(r, f_{j}\right)\right\}<\lambda T\left(r, f_{1}\right)+O\left(\log ^{+} T\left(r, f_{1}\right)\right)
$$

for all $r$ outside possibly a set of finite logarithmic measure, where $\lambda<1$ is a positive number. Then either $f_{2}=1$ or $f_{3}=1$.

Remark 3.2 Here $N_{2}\left(r, \frac{1}{f}\right)$ is the counting function of zeros of $f$ in $|z| \leq r$, where the simple zero is counted once, and the multiple zero is counted twice.

\section{The proof of Theorem 2.1}

Proof Let $f\left(z_{1}, z_{2}\right)$ be a transcendental finite-order entire solution of equation (2.1). We first rewrite (2.1) in the form

$$
\left(\frac{\frac{\partial^{2} f\left(z_{1}, z_{2}\right)}{\partial z_{1}^{2}}}{e^{\frac{g\left(z_{1}, z_{2}\right)}{2}}}\right)^{2}+\left(\frac{f\left(z_{1}+c_{1}, z_{2}+c_{2}\right)}{e^{\frac{g\left(z_{1}, z_{2}\right)}{2}}}\right)^{2}=1
$$


or

$$
\left(\frac{\frac{\partial^{2} f\left(z_{1}, z_{2}\right)}{\partial z_{1}^{2}}}{e^{\frac{g\left(z_{1}, z_{2}\right)}{2}}}+i \frac{f\left(z_{1}+c_{1}, z_{2}+c_{2}\right)}{e^{\frac{g\left(z_{1}, z_{2}\right)}{2}}}\right)\left(\frac{\frac{\partial^{2} f\left(z_{1}, z_{2}\right)}{\partial z_{1}^{2}}}{e^{\frac{g\left(z_{1}, z_{2}\right)}{2}}}-i \frac{f\left(z_{1}+c_{1}, z_{2}+c_{2}\right)}{e^{\frac{g\left(z_{1}, z_{2}\right)}{2}}}\right)=1 .
$$

Since $f$ is a finite-order transcendental entire function and $g$ is a polynomial, by Lemmas 3.1 and 3.2 there exists a polynomial $p(z)$ such that

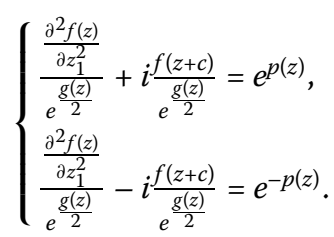

Denote

$$
\gamma_{1}(z)=\frac{g(z)}{2}+p(z), \quad \gamma_{2}(z)=\frac{g(z)}{2}-p(z)
$$

By combining with (4.2) it follows that

$$
\begin{gathered}
\frac{\partial^{2} f(z)}{\partial z_{1}^{2}}=\frac{e^{\gamma_{1}(z)}+e^{\gamma_{2}(z)}}{2}, \\
f(z+c)=\frac{e^{\gamma_{1}(z)}-e^{\gamma_{2}(z)}}{2 i} .
\end{gathered}
$$

This leads to

$$
-i Q_{1}(z) e^{\gamma_{1}(z)-\gamma_{1}(z+c)}+i Q_{2}(z) e^{\gamma_{2}(z)-\gamma_{1}(z+c)}-e^{\gamma_{2}(z+c)-\gamma_{1}(z+c)} \equiv 1
$$

where

$$
Q_{1}(z)=\frac{\partial^{2} \gamma_{1}}{\partial z_{1}^{2}}+\left(\frac{\partial \gamma_{1}}{\partial z_{1}}\right)^{2}, \quad Q_{2}(z)=\frac{\partial^{2} \gamma_{2}}{\partial z_{1}^{2}}+\left(\frac{\partial \gamma_{2}}{\partial z_{1}}\right)^{2}
$$

We consider two cases.

Case 1. If $e^{\gamma_{2}(z+c)-\gamma_{1}(z+c)}$ is a constant, then $\gamma_{2}(z+c)-\gamma_{1}(z+c)$ is a constant. Set $\gamma_{2}(z+$ $c)-\gamma_{1}(z+c)=\kappa, \kappa \in \mathbb{C}$. In view of (4.3), $p(z)$ is a constant. Let $\xi=e^{p(z)}$. Then equations (4.4) $-(4.5)$ can be represented as

$$
\frac{\partial^{2} f(z)}{\partial z_{1}^{2}}=K_{1} e^{\frac{g(z)}{2}}, \quad f(z+c)=K_{2} e^{\frac{g(z)}{2}}
$$

where $K_{1}=\frac{\xi+\xi^{-1}}{2}, K_{2}=\frac{\xi-\xi^{-1}}{2 i}$, and $K_{1}^{2}+K_{2}^{2}=1$.

This leads to

$$
\frac{K_{2}}{2 K_{1}}\left(\frac{\partial^{2} g}{\partial z_{1}^{2}}+\frac{1}{2}\left(\frac{\partial g}{\partial z_{1}}\right)^{2}\right)=e^{\frac{g(z+c)-g(z)}{2}}
$$


Since $g(z)$ is a polynomial, (4.8) implies that $g(z+c)-g(z)$ is a constant in $\mathbb{C}$. Otherwise, we obtain a contradiction from the fact that the left-hand side of this equation is not transcendental but the right-hand side is transcendental. Thus it follows that $g(z)=L(z)+H(s)+B$, where $L(z)=A_{1} z_{1}+A_{2} z_{2}, A_{1} \neq 0$, and $H(s)$ is a polynomial in $s$ in $\mathbb{C}, s=c_{2} z_{1}-c_{1} z_{2}$.

We will prove that $H(s) \equiv 0$. If $\operatorname{deg}_{s} H=n$, then equation (4.8) implies

$$
4 A_{1} c_{2} \frac{d H}{d s}+c_{2}^{2} \frac{d^{2} H}{d s^{2}}+2 c_{2}^{2}\left(\frac{d H}{d s}\right)^{2} \equiv \zeta_{0},
$$

that is,

$$
4 A_{1} c_{2} \frac{d H}{d s}+c_{2}^{2} \frac{d^{2} H}{d s^{2}} \equiv \zeta_{0}-2 c_{2}^{2}\left(\frac{d H}{d s}\right)^{2},
$$

where $\zeta_{0} \in \mathbb{C}$. By comparing the degree of $s$ in both sides of the above equation we have $2(n-1)=n-1$, that is, $n=1$. Thus the form of $L(z)+H(s)+B$ is still the linear form of $A_{1} z_{1}+A_{2} z_{2}+B$, which means that $H(s) \equiv 0$. Hence it follows that $g(z)=L(z)+B=$ $A_{1} z_{1}+A_{2} z_{2}+B$. By combining with (4.6)-(4.8) we conclude that

$$
\begin{aligned}
& f\left(z_{1}, z_{2}\right)=K_{2} e^{\frac{g(z-c)}{2}}=K_{2} e^{\frac{1}{2}\left[A_{1} z_{1}+A_{2} z_{2}+B-\left(A_{1} c_{1}+A_{2} c_{2}\right)\right]}, \\
& \frac{\xi^{2}-1}{4 i\left(\xi^{2}+1\right)} A_{1}^{2}=e^{\frac{1}{2}\left(A_{1} c_{1}+A_{2} c_{2}\right)},
\end{aligned}
$$

which implies that

$$
f\left(z_{1}, z_{2}\right)=\frac{2 K_{1}}{A_{1}^{2}} e^{\frac{1}{2}\left[A_{1} z_{1}+A_{2} z_{2}+B\right]}=\frac{\xi^{2}+1}{A_{1}^{2} \xi} e^{\frac{1}{2} g\left(z_{1}, z_{2}\right)} .
$$

This completes the proof of Theorem 2.1(i).

Case 2. $e^{\gamma_{2}(z+c)-\gamma_{1}(z+c)}$ is not a constant. Obviously, $Q_{1}(z) \equiv 0$ and $Q_{2}(z) \equiv 0$ cannot hold at the same time. Otherwise, it would follows from (4.6) that $e^{\gamma_{2}(z+c)-\gamma_{1}(z+c)}=-1$, a contradiction. If $Q_{1}(z) \equiv 0$ and $Q_{2}(z) \not \equiv 0$, then from (4.6) this yields that

$$
i Q_{2}(z) e^{\gamma_{2}(z)-\gamma_{1}(z+c)}-e^{\gamma_{2}(z+c)-\gamma_{1}(z+c)} \equiv 1
$$

Thus we conclude that $e^{\gamma_{2}(z)-\gamma_{1}(z+c)}$ is a nonconstant because $e^{\gamma_{2}(z+c)-\gamma_{1}(z+c)}$ is not a constant. Moreover, it follows that $e^{\gamma_{2}(z+c)-\gamma_{2}(z)}$ is not a constant. Otherwise, $\gamma_{2}(z+c)=\gamma_{2}(z)+\zeta$, where $\zeta \in \mathbb{C}$. Then from (4.10) we have $\left[i Q_{2}(z) e^{-\zeta}-1\right] e^{\gamma_{2}(z+c)-\gamma_{1}(z+c)} \equiv 1$, which is a contradiction with the nonconstant $e^{\gamma_{2}(z+c)-\gamma_{1}(z+c)}$. Thus (4.10) can be written in the form

$$
i Q_{2}(z) e^{\gamma_{2}(z)}-e^{\gamma_{2}(z+c)}-e^{\gamma_{1}(z+c)} \equiv 0
$$

By applying Lemma 3.3 for (4.11) we easily get a contradiction. If $Q_{2}(z) \equiv 0$ and $Q_{1}(z) \not \equiv 0$, by using the same argument as before, we can get a contradiction. Hence we have that $Q_{1}(z) \not \equiv 0$ and $Q_{2}(z) \not \equiv 0$.

Since $\gamma_{1}(z), \gamma_{2}(z)$ are polynomials and $e^{\gamma_{2}(z+c)-\gamma_{1}(z+c)}$ is not a constant, by applying Lemma 3.4 to (4.6) it follows that

$$
-i Q_{1}(z) e^{\gamma_{1}(z)-\gamma_{1}(z+c)} \equiv 1 \quad \text { or } \quad i Q_{2}(z) e^{\gamma_{2}(z)-\gamma_{1}(z+c)} \equiv 1
$$


Subcase 2.1. Suppose that $-i Q_{1}(z) e^{\gamma_{1}(z)-\gamma_{1}(z+c)} \equiv 1$. Then it follows from (4.6) that $i Q_{2}(z) e^{\gamma_{2}(z)-\gamma_{2}(z+c)} \equiv 1$. This means that $\gamma_{1}(z)-\gamma_{1}(z+c)=\zeta_{1}$ and $\gamma_{2}(z)-\gamma_{2}(z+c)=\zeta_{2}$, where $\zeta_{1}, \zeta_{2} \in \mathbb{C}$. Hence we have that $\gamma_{1}(z)=L_{1}(z)+H_{1}(s)+B_{1}$ and $\gamma_{2}(z)=L_{2}(z)+H_{2}(s)+B_{2}$, where $L_{j}(z)=A_{j 1} z_{1}+A_{j 2} z_{2}, H_{j}(s), j=1,2$, are polynomials in $s=c_{2} z_{1}-c_{1} z_{2}, A_{j 1}, A_{j 2}, B_{j} \in \mathbb{C}$, $j=1,2$. In view of the definitions of $Q_{1}, Q_{2}$, similarly to the argument in Case 1 , we can conclude that $H_{1}(s)=H_{2}(s) \equiv 0$. In addition, it follows that $L_{1}(z) \neq L_{2}(z)$. Otherwise, $\gamma_{2}(z+c)-\gamma_{1}(z+c)$ is a constant, which implies that $e^{\gamma_{2}(z+c)-\gamma_{1}(z+c)}$ is a constant, a contradiction. Substituting these into (4.12), we have

$$
i A_{11}^{2} e^{-L_{1}(c)}=i A_{11}^{2} e^{-\left(A_{11} c_{1}+A_{12} c_{2}\right)}=-1, \quad i A_{21}^{2} e^{-L_{2}(c)}=i A_{21}^{2} e^{-\left(A_{21} c_{1}+A_{22} c_{2}\right)}=1 .
$$

By combining with (4.5) we have

$$
f(z)=\frac{e^{L_{1}(z)+B_{1}-L_{1}(c)}-e^{L_{2}(z)+B_{2}-L_{2}(c)}}{2 i}=\frac{A_{21}^{2} e^{L_{1}(z)+B_{1}}+A_{11}^{2} e^{L_{2}(z)+B_{2}}}{2 A_{11}^{2} A_{21}^{2}} .
$$

From the definitions of $\gamma_{1}(z)$ and $\gamma_{2}(z)$ we can see that

$$
g(z)=\gamma_{1}(z)+\gamma_{2}(z)=L(z)+B
$$

where $L(z)=L_{1}(z)+L_{2}(z), B=B_{1}+B_{2}$.

Subcase 2.2. Suppose that $i Q_{2}(z) e^{\gamma_{2}(z)-\gamma_{1}(z+c)} \equiv 1$. Then it follows from (4.6) that $-i Q_{1}(z) e^{\gamma_{1}(z)-\gamma_{2}(z+c)} \equiv 1$. This means that $\gamma_{2}(z)-\gamma_{1}(z+c)=\zeta_{1}$ and $\gamma_{1}(z)-\gamma_{2}(z+c)=\zeta_{2}$, where $\zeta_{1}, \zeta_{2} \in \mathbb{C}$. Thus it follows that $\gamma_{1}(z+2 c)-\gamma_{1}(z)=-\zeta_{1}-\zeta_{2}$ and $\gamma_{2}(z+c)-\gamma_{2}(z)=$ $-\zeta_{1}-\zeta_{2}$. We can obtain that $\gamma_{1}(z)=L(z)+H(s)+B_{1}$ and $\gamma_{2}(z)=L(z)+H(s)+B_{2}$, where $L(z)=a_{1} z_{1}+a_{2} z_{2}$, and $H(s)$ is a polynomial in $s=c_{2} z_{1}-c_{1} z_{2}, a_{1}, a_{2}, B_{1}, B_{2} \in \mathbb{C}$. This yields that $\gamma_{2}(z+c)-\gamma_{1}(z+c)=B_{2}-B_{1}$, which implies that $e^{\gamma_{2}(z+c)-\gamma_{1}(z+c)}$ is a constant, a contradiction.

This completes the proof of Theorem 2.1.

\section{The proof of Theorem 2.2}

Proof Let $f\left(z_{1}, z_{2}\right)$ be a finite-order transcendental entire solution of equation (2.3). We first rewrite (2.3) in the form

$$
\left(\frac{\frac{\partial^{2} f\left(z_{1}, z_{2}\right)}{\partial z_{1}^{2}}}{e^{\frac{g\left(z_{1}, z_{2}\right)}{2}}}\right)^{2}+\left(\frac{f\left(z_{1}+c_{1}, z_{2}+c_{2}\right)-f\left(z_{1}, z_{2}\right)}{e^{\frac{g\left(z_{1}, z_{2}\right)}{2}}}\right)^{2}=1
$$

or

$$
\left(\frac{\frac{\partial^{2} f(z)}{\partial z_{1}^{2}}}{e^{\frac{g(z)}{2}}}+i \frac{f(z+c)-f(z)}{e^{\frac{g(z)}{2}}}\right)\left(\frac{\frac{\partial^{2} f(z)}{\partial z_{1}^{2}}}{e^{\frac{g(z)}{2}}}-i \frac{f(z+c)-f(z)}{e^{\frac{g(z)}{2}}}\right)=1 .
$$


Since $f$ is a finite-order transcendental entire function and $g$ is a polynomial, by Lemmas 3.1 and 3.2 there exists a polynomial $p(z)$ in $\mathbb{C}^{2}$ such that

$$
\left\{\begin{array}{l}
\frac{\frac{\partial^{2} f(z)}{\partial z_{1}^{2}}}{e^{\frac{g(z)}{2}}}+i \frac{f(z+c)-f(z)}{e^{\frac{g(z)}{2}}}=e^{p(z)}, \\
\frac{\frac{\partial^{2} f(z)}{\partial z_{1}^{2}}}{e^{\frac{g(z)}{2}}}-i \frac{f(z+c)-f(z)}{e^{\frac{g(z)}{2}}}=e^{-p(z)}
\end{array}\right.
$$

Denote

$$
\gamma_{1}(z)=\frac{g(z)}{2}+p(z), \quad \gamma_{2}(z)=\frac{g(z)}{2}-p(z)
$$

By combining with (5.2) it follows that

$$
\begin{gathered}
\frac{\partial^{2} f\left(z_{1}, z_{2}\right)}{\partial z_{1}^{2}}=\frac{e^{\gamma_{1}(z)}+e^{\gamma_{2}(z)}}{2}, \\
f(z+c)-f(z)=\frac{e^{\gamma_{1}(z)}-e^{\gamma_{2}(z)}}{2 i} .
\end{gathered}
$$

This leads to

$$
Q_{3}(z) e^{\gamma_{1}(z)-\gamma_{1}(z+c)}+Q_{4}(z) e^{\gamma_{2}(z)-\gamma_{1}(z+c)}-e^{\gamma_{2}(z+c)-\gamma_{1}(z+c)} \equiv 1,
$$

where

$$
Q_{3}(z)=1-i\left(\frac{\partial^{2} \gamma_{1}}{\partial z_{1}^{2}}+\left(\frac{\partial \gamma_{1}}{\partial z_{1}}\right)^{2}\right), \quad Q_{4}(z)=1+i\left(\frac{\partial^{2} \gamma_{2}}{\partial z_{1}^{2}}+\left(\frac{\partial \gamma_{2}}{\partial z_{1}}\right)^{2}\right) .
$$

We consider two cases.

Case 1. If $e^{\gamma_{2}(z+c)-\gamma_{1}(z+c)}$ is a constant, then $\gamma_{2}(z+c)-\gamma_{1}(z+c)$ is a constant. Set $\gamma_{2}(z+c)-$ $\gamma_{1}(z+c)=\kappa, \kappa \in \mathbb{C}$. In view of (5.3), this yields that $p(z)$ is a constant. Let $\xi=e^{p(z)}$. Then equations (5.4)-(5.5) can be represented as

$$
\frac{\partial^{2} f(z)}{\partial z_{1}^{2}}=K_{1} e^{\frac{g(z)}{2}}, \quad f(z+c)-f(z)=K_{2} e^{\frac{g(z)}{2}}
$$

where $K_{1}=\frac{\xi+\xi^{-1}}{2}, K_{2}=\frac{\xi-\xi^{-1}}{2 i}$, and $K_{1}^{2}+K_{2}^{2}=1$.

This leads to

$$
\frac{K_{2}}{2 K_{1}}\left(\frac{\partial^{2} g}{\partial z_{1}^{2}}+\frac{1}{2}\left(\frac{\partial g}{\partial z_{1}}\right)^{2}\right)+1=e^{\frac{g(z+c)-g(z)}{2}} .
$$

Since $g(z)$ is a polynomial, (5.8) implies $g(z+c)-g(z)$, and thus $e^{\frac{g(z+c)-g(z)}{2}}$ must be a constant. Denote $g(z+c)-g(z)=\zeta$, where $\zeta$ is a constant in $\mathbb{C}$. By using the same argument as in Case 1 of Theorem 2.1, we obtain that $g(z)=L(z)+B$, where $L(z)=A_{1} z_{1}+A_{2} z_{2}, B \in \mathbb{C}$.

By combining with (5.8) it follows that

$$
\frac{K_{2}}{4 K_{1}} A_{1}^{2}+1=e^{\frac{1}{2}\left(A_{1} c_{1}+A_{2} c_{2}\right)} .
$$


Solving the first equation in (5.7), we have

$$
\begin{aligned}
f\left(z_{1}, z_{2}\right) & =\frac{4 K_{1}}{A_{1}^{2}} e^{\frac{1}{2} g(z)}+z_{1} G_{1}\left(z_{2}\right)+G_{2}\left(z_{2}\right) \\
& =\frac{4\left(\xi^{2}+1\right)}{A_{1}^{2} \xi} e^{\frac{1}{2}(L(z)+B)}+z_{1} G_{1}\left(z_{2}\right)+G_{2}\left(z_{2}\right) .
\end{aligned}
$$

Substituting (5.10) into the second equation in (5.7) and combining with (5.9), we get that $G_{1}\left(z_{2}+c_{2}\right)=G_{1}\left(z_{2}\right)$ and $G_{2}\left(z_{2}+c_{2}\right)-G_{2}\left(z_{2}\right)=c_{1} G_{1}\left(z_{2}\right)$, which means that $G_{1}\left(z_{2}\right)$ is a finite-order entire period functions in $z_{2}$ with period $c_{2}$.

Case 2. $e^{\gamma_{2}(z+c)-\gamma_{1}(z+c)}$ is not a constant. Obviously, $Q_{3}(z) \equiv 0$ and $Q_{4}(z) \equiv 0$ cannot hold at the same time. Otherwise, it would follow from (5.6) that $e^{\gamma_{2}(z+c)-\gamma_{1}(z+c)}=-1$, a contradiction. If $Q_{3}(z) \equiv 0$ and $Q_{4}(z) \not \equiv 0$, then from (5.6) it follows that

$$
Q_{3}(z) e^{\gamma_{2}(z)-\gamma_{1}(z+c)}-e^{\gamma_{2}(z+c)-\gamma_{1}(z+c)} \equiv 1
$$

Thus we conclude that $e^{\gamma_{2}(z)-\gamma_{1}(z+c)}$ is not a constant because $e^{\gamma_{2}(z+c)-\gamma_{1}(z+c)}$ is not a constant. Moreover, it follows that $e^{\gamma_{2}(z+c)-\gamma_{2}(z)}$ is not a constant. Otherwise, $\gamma_{2}(z+c)=\gamma_{2}(z)+\zeta$, where $\zeta \in \mathbb{C}$. Then from (5.11) we have $\left[Q_{4}(z) e^{-\zeta}-1\right] e^{\gamma_{2}(z+c)-\gamma_{1}(z+c)} \equiv 1$, which is a contradiction with the nonconstant $e^{\gamma_{2}(z+c)-\gamma_{1}(z+c)}$. Thus (5.11) can be written in the form

$$
Q_{4}(z) e^{\gamma_{2}(z)}-e^{\gamma_{2}(z+c)}-e^{\gamma_{1}(z+c)} \equiv 0
$$

By applying Lemma 3.3 to (5.12) we easily get a contradiction. If $Q_{4}(z) \equiv 0$ and $Q_{3}(z) \not \equiv 0$, by using the same argument as before we can get a contradiction. Hence we have that $Q_{3}(z) \not \equiv 0$ and $Q_{4}(z) \not \equiv 0$.

Since $\gamma_{1}(z), \gamma_{2}(z)$ are polynomials and $e^{\gamma_{2}(z+c)-\gamma_{1}(z+c)}$ is a nonconstant, by applying Lemma 3.4 to (5.6) it follows that

$$
Q_{3}(z) e^{\gamma_{1}(z)-\gamma_{1}(z+c)} \equiv 1 \quad \text { or } \quad Q_{4}(z) e^{\gamma_{2}(z)-\gamma_{1}(z+c)} \equiv 1
$$

Subcase 2.1. Suppose that $Q_{3}(z) e^{\gamma_{1}(z)-\gamma_{1}(z+c)} \equiv 1$. Then it follows from (5.6) that $Q_{4}(z) e^{\gamma_{2}(z)-\gamma_{2}(z+c)} \equiv 1$. This means that $\gamma_{1}(z)-\gamma_{1}(z+c)=\zeta_{1}, \gamma_{2}(z)-\gamma_{2}(z+c)=\zeta_{2}$, where $\zeta_{1}, \zeta_{2} \in \mathbb{C}$. Hence we have that $\gamma_{1}(z)=L_{1}(z)+H_{1}(s)+B_{1}$ and $\gamma_{2}(z)=L_{2}(z)+H_{2}(s)+B_{2}$, where $L_{j}(z)=A_{j 1} z_{1}+A_{j 2} z_{2}, H_{j}\left(s_{1}\right), j=1,2$, are polynomials in $s_{1}=c_{2} z_{1}-c_{1} z_{2}, A_{j 1}, A_{j 2}, B_{j} \in \mathbb{C}$, $j=1$, 2. Similarly to the argument in Case 1 of Theorem 2.2, we have $H_{1}(s)=H_{2}(s) \equiv 0$. Thus it follows that $\gamma_{1}(z)=L_{1}(z)+B_{1}$ and $\gamma_{2}(z)=L_{2}(z)+B_{2}$. Obviously, $L_{1}(z) \neq L_{2}(z)$. Otherwise, $\gamma_{2}(z+c)-\gamma_{1}(z+c)$ is a constant, which implies that $e^{\gamma_{2}(z+c)-\gamma_{1}(z+c)}$ is a constant, a contradiction. Substituting these into (5.6), we have

$$
\left(1-i A_{11}^{2}\right) e^{-\left(A_{11} c_{1}+A_{12} c_{2}\right)}=1, \quad\left(1+i A_{21}^{2}\right) e^{-\left(A_{21} c_{1}+A_{22} c_{2}\right)}=1 .
$$

By solving the equation

$$
\frac{\partial^{2} f(z)}{\partial z_{1}^{2}}=\frac{e^{L_{1}(z)+B_{1}}+e^{L_{2}(z)+B_{2}}}{2}
$$


we have

$$
f\left(z_{1}, z_{2}\right)=\frac{A_{21}^{2} e^{L_{1}(z)+B_{1}}+A_{11}^{2} e^{L_{2}(z)+B_{2}}}{2 A_{11}^{2} A_{21}^{2}}+z_{1} G_{1}\left(z_{2}\right)+G_{2}\left(z_{2}\right) .
$$

Substituting (5.16) into (5.5) and combining with (5.14), we have $G_{1}\left(z_{2}+c_{2}\right)=G_{1}\left(z_{2}\right)$ and $G_{2}\left(z_{2}+c_{2}\right)-G_{2}\left(z_{2}\right)=c_{1} G_{1}\left(z_{2}\right)$, which means that $G_{1}\left(z_{2}\right)$ is a finite-order entire periodic function in $z_{2}$ with period $c_{2}$.

From the definitions of $\gamma_{1}(z)$ and $\gamma_{2}(z)$ we can see that

$$
g(z)=\gamma_{1}(z)+\gamma_{2}(z)=L(z)+B,
$$

where $L(z)=L_{1}(z)+L_{2}(z), B=B_{1}+B_{2}$.

Subcase 2.2. Suppose that $Q_{4}(z) e^{\gamma_{2}(z)-\gamma_{1}(z+c)} \equiv 1$. Similarly to the argument in Subcase 2.2 in Theorem 2.1, we can get a contradiction.

Therefore this completes the proof of Theorem 2.2.

\section{Proofs of Theorems 2.3 and 2.4}

\subsection{Proof of Theorem 2.4}

Suppose that $f\left(z_{1}, z_{2}\right)$ is a finite-order transcendental entire solution of equation (2.7). We first rewrite (2.7) in the form

$$
\left(\frac{\frac{\partial^{2} f(z)}{\partial z_{1} \partial \partial 2}}{e^{\frac{g(z)}{2}}}+i \frac{f(z+c)-f(z)}{e^{\frac{g(z)}{2}}}\right)\left(\frac{\frac{\partial^{2} f(z)}{\partial z^{1} \partial z_{2}}}{e^{\frac{g(z)}{2}}}-i \frac{f(z+c)-f(z)}{e^{\frac{g(z)}{2}}}\right)=1 .
$$

Since $f$ is a finite-order transcendental entire function and $g$ is a polynomial, by Lemmas 3.1 and 3.2 there exists a polynomial $p(z)$ such that

$$
\left\{\begin{array}{l}
\frac{\partial^{2} f(z)}{\frac{\partial z_{1}(g z 2}{e^{\frac{g}{2}(z)}}}+i \frac{f(z+c)-f(z)}{e^{\frac{g(z)}{2}}}=e^{p(z)}, \\
\frac{\frac{\partial^{2} f(z)}{\partial z_{1} z_{2}}}{e^{\frac{g(z)}{2}}}-i \frac{f(z+c)-f(z)}{e^{\frac{g(z)}{2}}}=e^{-p(z)} .
\end{array}\right.
$$

Denote

$$
\gamma_{1}(z)=\frac{g(z)}{2}+p(z), \quad \gamma_{2}(z)=\frac{g(z)}{2}-p(z) .
$$

By combining with (6.2) it follows that

$$
\begin{gathered}
\frac{\partial^{2} f\left(z_{1}, z_{2}\right)}{\partial z_{1} \partial z_{2}}=\frac{e^{\gamma_{1}(z)}+e^{\gamma_{2}(z)}}{2}, \\
f(z+c)-f(z)=\frac{e^{\gamma_{1}(z)}-e^{\gamma_{2}(z)}}{2 i} .
\end{gathered}
$$

This leads to

$$
Q_{5}(z) e^{\gamma_{1}(z)-\gamma_{1}(z+c)}+Q_{6}(z) e^{\gamma_{2}(z)-\gamma_{1}(z+c)}-e^{\gamma_{2}(z+c)-\gamma_{1}(z+c)} \equiv 1,
$$


where

$$
Q_{5}(z)=1-i\left(\frac{\partial^{2} \gamma_{1}}{\partial z_{1} \partial z_{2}}+\frac{\partial \gamma_{1}}{\partial z_{1}} \frac{\partial \gamma_{1}}{\partial z_{2}}\right), \quad Q_{4}(z)=1+i\left(\frac{\partial^{2} \gamma_{2}}{\partial z_{1} \partial z_{2}}+\frac{\partial \gamma_{2}}{\partial z_{1}} \frac{\partial \gamma_{2}}{\partial z_{2}}\right)
$$

We consider two cases.

Case 1. If $e^{\gamma_{2}(z+c)-\gamma_{1}(z+c)}$ is a constant, then $\gamma_{2}(z+c)-\gamma_{1}(z+c)$ is a constant. Set $\gamma_{2}(z+c)-$ $\gamma_{1}(z+c)=\kappa, \kappa \in \mathbb{C}$. In view of (6.3), this yields that $p(z)$ is a constant. Let $\xi=e^{p(z)}$. Then equations (6.4)-(6.5) become

$$
\frac{\partial^{2} f(z)}{\partial z_{1} \partial z_{2}}=K_{1} e^{\frac{g(z)}{2}}, \quad f(z+c)-f(z)=K_{2} e^{\frac{g(z)}{2}},
$$

where $K_{1}=\frac{\xi+\xi^{-1}}{2}, K_{2}=\frac{\xi-\xi^{-1}}{2 i}$, and $K_{1}^{2}+K_{2}^{2}=1$.

This leads to

$$
\frac{K_{2}}{2 K_{1}}\left(\frac{\partial^{2} g}{\partial z_{1} \partial z_{2}}+\frac{1}{2} \frac{\partial g}{\partial z_{1}} \frac{\partial g}{\partial z_{2}}\right)+1=e^{\frac{g(z+c)-g(z)}{2}}
$$

Since $g(z)$ is a polynomial, (6.8) implies $g(z+c)-g(z)$, and thus $e^{\frac{g(z+c)-g(z)}{2}}$ must be a constant. Denote $g(z+c)-g(z)=\zeta$, where $\zeta$ is a constant in $\mathbb{C}$. Thus it follows that $g(z)=L(z)+H(s)+$ $B$, where $L(z)=A_{1} z_{1}+A_{2} z_{2}$, and $H(s)$ is a polynomial in $s$ in $\mathbb{C}, s=c_{2} z_{1}-c_{1} z_{2}$. Substituting this into (6.8), we deduce that

$$
\frac{K_{2}}{2 K_{1}}\left(-\frac{1}{4} c_{1} c_{2} H^{\prime \prime}-\frac{1}{2} c_{1} c_{2}\left(H^{\prime}\right)^{2}+\frac{1}{2}\left(A_{2} c_{2}-A_{1} c_{1}\right) H^{\prime}+\frac{1}{2} A_{1} A_{2}\right)+1=e^{\frac{g(z+c)-g(z)}{2}} .
$$

Since $g(z)$ is a polynomial, then (6.9) implies that $g(z+c)-g(z)$ is a constant in $\mathbb{C}$. Otherwise, we would obtain a contradiction from the fact that the left-hand side of the above equation is not transcendental but the right-hand side is transcendental. Hence it follows that

$$
\frac{K_{2}}{2 K_{1}}\left(-\frac{1}{4} c_{1} c_{2} H^{\prime \prime}-\frac{1}{2} c_{1} c_{2}\left(H^{\prime}\right)^{2}+\frac{1}{2}\left(A_{2} c_{2}-A_{1} c_{1}\right) H^{\prime}+\frac{1}{2} A_{1} A_{2}\right)+1=\zeta_{0},
$$

where $\zeta_{0} \in \mathbb{C}$. If $c_{1}=0, c_{2} \neq 0$, that is, $\frac{K_{2}}{2 K_{1}}\left(\frac{1}{2} A_{2} c_{2} H^{\prime}+\frac{1}{2} A_{1} A_{2}\right)+1=\zeta_{0}$. Thus, either $A_{2}=0$, or $H^{\prime}$ is a constant. If $A_{2}=0$, then $\zeta_{0}=1$, that is, $e^{\frac{g(z+c)-g(z)}{2}}$ is a constant. By combining with $c_{1}=0$ this means that $g(z)$ is a constant. Set $e^{\frac{g}{2}}=\theta$. In view of the first equation of (6.5), we have

$$
f\left(z_{1}, z_{2}\right)=K_{1} \theta z_{1} z_{2}+\mu\left(z_{1}\right),
$$

where $\mu\left(z_{1}\right)$ is a finite-order transcendental entire function. Substituting this into the second equation of (6.5), we have

$$
K_{1} \theta\left(z_{1}+c_{1}\right)\left(z_{2}+c_{2}\right)+\mu\left(z_{1}+c_{1}\right)-K_{1} \theta z_{1} z_{2}-\mu\left(z_{1}\right)=K_{2} \theta .
$$

Combining with $c_{1}=0$, this yields that $K_{1} \theta c_{2} z_{1}=K_{2} \theta$, which is impossible. Hence $H^{\prime}$ is a constant, that is, $H(s)=c 2 z_{1}$. 
If $c_{2}=0, c_{1} \neq 0$, similarly to the above argument, we can obtain that $H(s)=-c_{1} z_{2}$.

Let $c_{1} \neq 0$ and $c_{2} \neq 0$. If $A_{2} c_{2}-A_{1} c_{1}=0$, noting that the left-hand side of (6.10) is a constant, we have $\operatorname{deg}_{s} H \leq 1$, that is, $H(s)=c_{2} z_{1}-c_{1} z_{2}+\tau$, where $\tau \in \mathbb{C}$. If $A_{2} c_{2}-A_{1} c_{1} \neq 0$, we easily obtain that $\operatorname{deg}_{s} H \leq 1$, that is, $H(s)=c_{2} z_{1}-c_{1} z_{2}++\tau$, where $\tau \in \mathbb{C}$. Thus the form of $L(z)+H(s)+B$ is still the linear form of $A_{1} z_{1}+A_{2} z_{2}+B$, which means that $H(s) \equiv 0$. Hence we obtain that $g(z)=L(z)+B$, where $L(z)=A_{1} z_{1}+A_{2} z_{2}, B \in \mathbb{C}$.

By combining with (6.8) it follows that

$$
\frac{K_{2}}{4 K_{1}} A_{1} A_{2}+1=e^{\frac{1}{2}\left(A_{1} c_{1}+A_{2} c_{2}\right)} .
$$

Solving the first equation in (6.7), we have

$$
\begin{aligned}
f\left(z_{1}, z_{2}\right) & =\frac{4 K_{1}}{A_{1} A_{2}} e^{\frac{1}{2} g(z)}+\phi\left(z_{1}\right)+\varphi\left(z_{2}\right) \\
& =\frac{4\left(\xi^{2}+1\right)}{A_{1} A_{2} \xi} e^{\frac{1}{2}(L(z)+B)}+\phi\left(z_{1}\right)+\varphi\left(z_{2}\right) .
\end{aligned}
$$

Substituting (6.13) into the second equation in (6.7) and combining with (6.12), we get that

$$
\phi\left(z_{1}+c_{1}\right)-\phi\left(z_{1}\right)=-\left[\varphi\left(z_{2}+c_{2}\right)-\varphi\left(z_{2}\right)\right]
$$

which yields that $\phi\left(z_{1}\right)=G_{3}\left(z_{1}\right)+D_{1} z_{1}$ and $\varphi\left(z_{2}\right)=G_{4}\left(z_{2}\right)+D_{2} z_{2}$, where $D_{1} c_{1}+D_{2} c_{2}=0$ and $G_{3}\left(z_{1}\right), G_{4}\left(z_{2}\right)$ are finite-order entire period functions in $z_{1}, z_{2}$ with periods $c_{1}, c_{2}$, respectively.

Case 2. $e^{\gamma_{2}(z+c)-\gamma_{1}(z+c)}$ is not a constant. Obviously, $Q_{5}(z) \equiv 0$ and $Q_{6}(z) \equiv 0$ cannot hold at the same time. Otherwise, it would follow from (6.6) that $e^{\gamma_{2}(z+c)-\gamma_{1}(z+c)}=-1$, a contradiction. If $Q_{5}(z) \equiv 0$ and $Q_{6}(z) \not \equiv 0$, then from (6.6) we get that

$$
Q_{5}(z) e^{\gamma_{2}(z)-\gamma_{1}(z+c)}-e^{\gamma_{2}(z+c)-\gamma_{1}(z+c)} \equiv 1
$$

Thus we conclude that $e^{\gamma_{2}(z)-\gamma_{1}(z+c)}$ is not a constant because $e^{\gamma_{2}(z+c)-\gamma_{1}(z+c)}$ is not a constant. Moreover, it follows that $e^{\gamma_{2}(z+c)-\gamma_{2}(z)}$ is not a constant. Otherwise, $\gamma_{2}(z+c)=\gamma_{2}(z)+\zeta$, where $\zeta \in \mathbb{C}$. Then from (6.14) we have $\left[Q_{6}(z) e^{-\zeta}-1\right] e^{\gamma_{2}(z+c)-\gamma_{1}(z+c)} \equiv 1$, which is a contradiction with the nonconstant $e^{\gamma_{2}(z+c)-\gamma_{1}(z+c)}$. Thus (6.14) can be written in the form

$$
Q_{6}(z) e^{\gamma_{2}(z)}-e^{\gamma_{2}(z+c)}-e^{\gamma_{1}(z+c)} \equiv 0
$$

By applying Lemma 3.3 for (6.15) we easily get a contradiction. If $Q_{6}(z) \equiv 0$ and $Q_{5}(z) \not \equiv 0$, by using the same argument as before we can get a contradiction. Hence we have that $Q_{5}(z) \not \equiv 0$ and $Q_{6}(z) \not \equiv 0$.

Since $\gamma_{1}(z), \gamma_{2}(z)$ are polynomials and $e^{\gamma_{2}(z+c)-\gamma_{1}(z+c)}$ is not a constant, by applying Lemma 3.4 to (6.6) it follows that

$$
Q_{5}(z) e^{\gamma_{1}(z)-\gamma_{1}(z+c)} \equiv 1 \quad \text { or } \quad Q_{6}(z) e^{\gamma_{2}(z)-\gamma_{1}(z+c)} \equiv 1
$$


Subcase 2.1. Suppose that $Q_{5}(z) e^{\gamma_{1}(z)-\gamma_{1}(z+c)} \equiv 1$. Then it follows from (6.6) that $Q_{6}(z) e^{\gamma_{2}(z)-\gamma_{2}(z+c)} \equiv 1$. This means that $\gamma_{1}(z)-\gamma_{1}(z+c)=\zeta_{1}, \gamma_{2}(z)-\gamma_{2}(z+c)=\zeta_{2}$, where $\zeta_{1}, \zeta_{2} \in \mathbb{C}$. Hence we have that $\gamma_{1}(z)=L_{1}(z)+H_{1}(s)+B_{1}$ and $\gamma_{2}(z)=L_{2}(z)+H_{2}(s)+B_{2}$, where $L_{j}(z)=A_{j 1} z_{1}+A_{j 2} z_{2}, H_{j}(s), j=1,2$, are polynomials in $s=c_{2} z_{1}-c_{1} z_{2}, A_{j 1}, A_{j 2}, B_{j} \in \mathbb{C}$, $j=1,2$. Similarly to the argument in Case 1 , we have $H_{1}(s)=H_{2}(s) \equiv 0$. Thus it follows that $\gamma_{1}(z)=L_{1}(z)+B_{1}$ and $\gamma_{2}(z)=L_{2}(z)+B_{2}$. Obviously, $L_{1}(z) \neq L_{2}(z)$. Otherwise, $\gamma_{2}(z+c)-\gamma_{1}(z+c)$ would be a constant, which implies that $e^{\gamma_{2}(z+c)-\gamma_{1}(z+c)}$ is a constant, a contradiction. Substituting these into (6.6), we have

$$
\left(1-i A_{11} A_{12}\right) e^{-\left(A_{11} c_{1}+A_{12} c_{2}\right)}=1, \quad\left(1+i A_{21} A_{22}\right) e^{-\left(A_{21} c_{1}+A_{22} c_{2}\right)}=1 .
$$

By solving the equation

$$
\frac{\partial^{2} f(z)}{\partial z_{1} \partial z_{2}}=\frac{e^{L_{1}(z)+B_{1}}+e^{L_{2}(z)+B_{2}}}{2}
$$

we have

$$
f\left(z_{1}, z_{2}\right)=\frac{e^{L_{1}(z)+B_{1}}}{2 A_{11} A_{12}}+\frac{e^{L_{2}(z)+B_{2}}}{2 A_{21} A_{22}}+\phi\left(z_{1}\right)+\varphi\left(z_{2}\right) .
$$

Substituting (6.19) into (6.5) and combining with (6.17), we get that

$$
\phi\left(z_{1}+c_{1}\right)-\phi\left(z_{1}\right)=-\left[\varphi\left(z_{2}+c_{2}\right)-\varphi\left(z_{2}\right)\right],
$$

which yields that $\phi\left(z_{1}\right)=G_{3}\left(z_{1}\right)+D_{1} z_{1}$ and $\varphi\left(z_{2}\right)=G_{4}\left(z_{2}\right)+D_{2} z_{2}$, where $D_{1} c_{1}+D_{2} c_{2}=0$ and $G_{3}\left(z_{1}\right), G_{4}\left(z_{2}\right)$ are finite-order entire periodic functions in $z_{1}, z_{2}$ with period $c_{1}, c_{2}$, respectively.

From the definitions of $\gamma_{1}(z)$ and $\gamma_{2}(z)$ we can see that

$$
g(z)=\gamma_{1}(z)+\gamma_{2}(z)=L(z)+B
$$

where $L(z)=L_{1}(z)+L_{2}(z), B=B_{1}+B_{2}$.

Subcase 2.2. Suppose that $Q_{6}(z) e^{\gamma_{2}(z)-\gamma_{1}(z+c)} \equiv 1$. Similarly to the argument in Subcase 2.2 in Theorem 2.1, we can get a contradiction.

This completes the proof of Theorem 2.4.

\subsection{Proof of Theorem 2.3}

Similar to the argument in the proof of Theorem 2.1, we can easily prove the statements of Theorem 2.3.

\section{Remarks}

In view of the arguments in the proofs of Theorems 2.1 and 2.3, we easily get the following theorems.

Theorem 7.1 Let $c=\left(c_{1}, c_{2}\right) \in \mathbb{C}^{2}$ with $c_{1} \neq 0, c_{2} \neq 0$, and $c_{1}+c_{2} \neq 0$. If the partial differential-difference equation

$$
\left(\frac{\partial^{2} f\left(z_{1}, z_{2}\right)}{\partial z_{1}^{2}}+\frac{\partial^{2} f\left(z_{1}, z_{2}\right)}{\partial z_{1} \partial z_{2}}\right)^{2}+f\left(z_{1}+c_{1}, z_{2}+c_{2}\right)^{2}=e^{g\left(z_{1}, z_{2}\right)}
$$


admits a transcendental entire solution $f\left(z_{1}, z_{2}\right)$ of finite order, then $g(z)$ must be a polynomial function of the form $g(z)=L(z)+B$, where $L(z)$ is a linear function of the form $L(z)=A_{1} z_{1}+A_{2} z_{2}+B, A_{1}, A_{2}, B \in \mathbb{C}$. Further, $f\left(z_{1}, z_{2}\right)$ must satisfy one of the following cases:

(i)

$$
f\left(z_{1}, z_{2}\right)=\frac{4\left(\xi^{2}+1\right)}{A_{1}\left(A_{1}+A_{2}\right) \xi} e^{\frac{1}{2} g\left(z_{1}, z_{2}\right)}
$$

with $\xi(\neq 0), A_{1}, A_{2}, B \in \mathbb{C}$ satisfying

$$
\frac{1}{4} \frac{\xi^{2}-1}{\left(\xi^{2}+1\right) i} A_{1}\left(A_{1}+A_{2}\right)=e^{\frac{1}{2}\left(A_{1} c_{1}+A_{2} c_{2}\right)}
$$

(ii)

$$
f\left(z_{1}, z_{2}\right)=\frac{e^{L_{1}(z)+B_{1}}}{2 A_{11}\left(A_{11}+A_{12}\right)}+\frac{e^{L_{2}(z)+B_{2}}}{2 A_{21}\left(A_{21}+A_{12}\right)},
$$

where $L_{1}(z)=A_{11} z_{1}+A_{12} z_{2}+B_{1}, L_{2}(z)=A_{21} z_{1}+A_{22} z_{2}+B_{2}, A_{j 1}, A_{j 2}, B_{j} \in \mathbb{C}(j=1,2)$ satisfy

$$
L_{1}(z) \neq L_{2}(z), \quad g(z)=L_{1}(z)+L_{2}(z)+B_{1}+B_{2}
$$

and

$$
-i A_{11}\left(A_{11}+A_{12}\right) e^{-L_{1}(c)}=i A_{21}\left(A_{21}+A_{22}\right) e^{-L_{2}(c)}=1
$$

We give some examples showing the existence of finite-order transcendental entire solutions of equation (7.1).

Example 7.1 Let $A_{1}=1, A_{2}=1, B=0$, and

$$
f\left(z_{1}, z_{2}\right)=\frac{2 \sqrt{5}}{5} e^{\frac{1}{2}\left(z_{1}+z_{2}\right)} .
$$

Then $\rho(f)=1$, and $f\left(z_{1}, z_{2}\right)$ is a transcendental entire solution of equation (7.1) with $g(z)=$ $z_{1}+z_{2}, c_{1}=2 \pi i$, and $c_{2}=2 \pi i$.

Example 7.2 Let $L_{1}(z)=i z_{1}+(1-i) z_{2}, L_{2}(z)=z_{1}+(i-1) z_{2}, B_{1}=B_{2}=0$, and

$$
f\left(z_{1}, z_{2}\right)=\frac{e^{i z_{1}+(1-i) z_{2}}}{2 i}+\frac{e^{z_{1}+(i-1) z_{2}}}{2 i} .
$$

Then $\rho(f)=1$, and $f\left(z_{1}, z_{2}\right)$ is a transcendental entire solution of equation (7.1) with $g(z)=$ $(1+i) z_{1}, c_{1}=\frac{3 \pi}{2}(1+i)$, and $c_{2}=\frac{\pi}{2}(1+2 i)$.

Theorem 7.2 Let $c=\left(c_{1}, c_{2}\right) \in \mathbb{C}^{2}$ with $c_{1} \neq 0, c_{2} \neq 0$, and $c_{1}+c_{2} \neq 0$. If the partial differential-difference equation

$$
\left(\frac{\partial^{2} f\left(z_{1}, z_{2}\right)}{\partial z_{1}^{2}}+\frac{\partial^{2} f\left(z_{1}, z_{2}\right)}{\partial z_{2}^{2}}\right)^{2}+f\left(z_{1}+c_{1}, z_{2}+c_{2}\right)^{2}=e^{g\left(z_{1}, z_{2}\right)}
$$


admits a transcendental entire solution $f\left(z_{1}, z_{2}\right)$ of finite order, then $g(z)$ must be a polynomial function of the form $g(z)=L(z)+B$, where $L(z)$ is a linear function of the form $L(z)=A_{1} z_{1}+A_{2} z_{2}+B, A_{1}, A_{2}, B \in \mathbb{C}$. Further, $f\left(z_{1}, z_{2}\right)$ must satisfy one of the following cases:

(i)

$$
f\left(z_{1}, z_{2}\right)=\frac{4\left(\xi^{2}+1\right)}{\left(A_{1}^{2}+A_{2}^{2}\right) \xi} e^{\frac{1}{2} g\left(z_{1}, z_{2}\right)}
$$

with $\xi(\neq 0), A_{1}, A_{2}, B \in \mathbb{C}$ satisfying

$$
\frac{1}{4} \frac{\xi^{2}-1}{\left(\xi^{2}+1\right) i}\left(A_{1}^{2}+A_{2}^{2}\right)=e^{\frac{1}{2}\left(A_{1} c_{1}+A_{2} c_{2}\right)}
$$

(ii)

$$
f\left(z_{1}, z_{2}\right)=\frac{e^{L_{1}(z)+B_{1}}}{2\left(A_{11}^{2}+A_{12}^{2}\right)}+\frac{e^{L_{2}(z)+B_{2}}}{2\left(A_{21}^{2}+A_{12}^{2}\right)},
$$

where $L_{1}(z)=A_{11} z_{1}+A_{12} z_{2}+B_{1}, L_{2}(z)=A_{21} z_{1}+A_{22} z_{2}+B_{2}, A_{j 1}, A_{j 2}, B_{j} \in \mathbb{C}(j=1,2)$ satisfy

$$
L_{1}(z) \neq L_{2}(z), \quad g(z)=L_{1}(z)+L_{2}(z)+B_{1}+B_{2}
$$

and

$$
-i\left(A_{11}^{2}+A_{12}^{2}\right) e^{-L_{1}(c)}=i\left(A_{21}^{2}+A_{22}^{2}\right) e^{-L_{2}(c)}=1
$$

Some examples explain the existence of finiteorder - entire solutions of equation (7.2).

Example 7.3 Let $A_{1}=1, A_{2}=1, B=0$, and

$$
f\left(z_{1}, z_{2}\right)=\frac{2 \sqrt{5}}{5} e^{\frac{1}{2}\left(z_{1}+z_{2}\right)} .
$$

Then $\rho(f)=1$, and $f\left(z_{1}, z_{2}\right)$ is a transcendental entire solution of equation (7.2) with $g(z)=$ $z_{1}+z_{2}, c_{1}=\pi i$, and $c_{2}=\pi i$.

Example 7.4 Let $L_{1}(z)=i z_{1}+\sqrt{2} z_{2}, L_{2}(z)=\sqrt{2} i z_{1}+z_{2}, B_{1}=B_{2}=0$, and

$$
f\left(z_{1}, z_{2}\right)=\frac{e^{i z_{1}+\sqrt{2} z_{2}}}{2}-\frac{e^{i z_{1}+\sqrt{2} z_{2}}}{2} .
$$

Then $\rho(f)=1$ and $f\left(z_{1}, z_{2}\right)$ is a transcendental entire solution of equation (7.2) with $g(z)=$ $(\sqrt{2}+1) i z_{1}+(\sqrt{2}+1) z_{2}, c_{1}=\frac{(\sqrt{2}-3) \pi}{2}$, and $c_{2}=\frac{(3 \sqrt{2}-1)}{2} \pi i$.

In view of Theorems 7.1 and 7.2, we easily get the following. 
Corollary 7.1 Let $c=\left(c_{1}, c_{2}\right) \in \mathbb{C}^{2}, c_{1} \neq 0, c_{2} \neq 0, \alpha, \beta, \gamma \in \mathbb{C}$, and let $g\left(z_{1}, z_{2}\right)$ be not a linear function of the form $L(z)=A_{1} z_{1}+A_{2} z_{2}+B$, where $A_{1}, A_{2}, B \in \mathbb{C}$. If $\alpha c_{1}^{2}-\beta c_{1} c_{2}+\gamma c_{2}^{2} \neq 0$, then the partial differential-difference equation

$$
\left(\alpha \frac{\partial^{2} f\left(z_{1}, z_{2}\right)}{\partial z_{1}^{2}}+\beta \frac{\partial^{2} f\left(z_{1}, z_{2}\right)}{\partial z_{1} \partial z_{2}}+\gamma \frac{\partial^{2} f\left(z_{1}, z_{2}\right)}{\partial z_{2}^{2}}\right)^{2}+f\left(z_{1}+c_{1}, z_{2}+c_{2}\right)^{2}=e^{g\left(z_{1}, z_{2}\right)}
$$

has no finite-order transcendental entire solution.

Corollary 7.2 The finite-order transcendental entire solution $f\left(z_{1}, z_{2}\right)$ of the partial differential equations

$$
\begin{aligned}
& \left(\frac{\partial^{2} f\left(z_{1}, z_{2}\right)}{\partial z_{1}^{2}}+\frac{\partial^{2} f\left(z_{1}, z_{2}\right)}{\partial z_{1} \partial z_{2}}\right)^{2}+f\left(z_{1}, z_{2}\right)^{2}=1 \\
& \left(\frac{\partial^{2} f\left(z_{1}, z_{2}\right)}{\partial z_{1}^{2}}+\frac{\partial^{2} f\left(z_{1}, z_{2}\right)}{\partial z_{2}^{2}}\right)^{2}+f\left(z_{1}, z_{2}\right)^{2}=1
\end{aligned}
$$

must be of the form

$$
f\left(z_{1}, z_{2}\right)=\frac{e^{L(z)+B}-e^{-L(z)-B}}{2 i}=\sin (-i(L(z)+B)),
$$

where $L(z)=A_{1} z_{1}+A_{2} z_{2}, A_{1}, A_{2}, B \in \mathbb{C}$ satisfy $A_{1}^{2}\left(A_{1}+A_{2}\right)^{2}=1$ and $\left(A_{1}^{2}+A_{2}^{2}\right)^{2}=1$.

Corresponding to Theorems 7.1 and 7.2, we can obtain some results on the existence of solutions of the difference-type equations (7.1) and (7.2).

Theorem 7.3 Let $c=\left(c_{1}, c_{2}\right) \in \mathbb{C}^{2}, c_{2} \neq 0, c_{1} \neq c_{2}$. If the partial differential-difference equation

$$
\left(\frac{\partial^{2} f\left(z_{1}, z_{2}\right)}{\partial z_{1}^{2}}+\frac{\partial^{2} f\left(z_{1}, z_{2}\right)}{\partial z_{1} \partial z_{2}}\right)^{2}+\left[f\left(z_{1}+c_{1}, z_{2}+c_{2}\right)-f\left(z_{1}, z_{2}\right)\right]^{2}=e^{g\left(z_{1}, z_{2}\right)}
$$

admits a transcendental entire solution of finite order, then $g\left(z_{1}, z_{2}\right)$ must be a linear function of the form $g\left(z_{1}, z_{2}\right)=A_{1} z_{1}+A_{2} z_{2}+B$, where $A_{1}, A_{2}, B \in \mathbb{C}$.

Theorem 7.4 Let $c=\left(c_{1}, c_{2}\right)$ be a constant in $\mathbb{C}^{2}$ such that $c_{1} \neq \pm i c_{2}$. If the partial differential-difference equation

$$
\left(\frac{\partial^{2} f\left(z_{1}, z_{2}\right)}{\partial z_{1}^{2}}+\frac{\partial^{2} f\left(z_{1}, z_{2}\right)}{\partial z_{2}^{2}}\right)^{2}+\left[f\left(z_{1}+c_{1}, z_{2}+c_{2}\right)-f\left(z_{1}, z_{2}\right)\right]^{2}=e^{g\left(z_{1}, z_{2}\right)}
$$

admits a transcendental entire solution of finite order, then $g\left(z_{1}, z_{2}\right)$ must be a linear function of the form $g\left(z_{1}, z_{2}\right)=A_{1} z_{1}+A_{2} z_{2}+B$, where $A_{1}, A_{2}, B \in \mathbb{C}$.

Remark 7.1 Although we give the conditions for the existence of finite-order transcendental entire solutions of equations (7.3) and (7.4) in Theorems 7.3 and 7.4, in view of Theorems 2.2 and 2.4, there naturally arises an open question: How to describe the forms of finite-order transcendental entire solutions of equations (7.3) and (7.4)? 


\section{Acknowledgements}

We thank the referee(s) for reading the manuscript very carefully and making a number of valuable and kind comments, which improved the presentation.

\section{Funding}

This work was supported by the National Natural Science Foundation of China $(11561033,61877046)$, the Natural Science Foundation of Jiangxi Province in China (20181BAB201001), Shangrao Science and Technology Talent Plan (2020K006) and the Foundation of Education Department of Jiangxi (GJJ190876, GJJ202303, GJJ191042, GJJ190895) of China.

\section{Availability of data and materials}

No data were used to support this study.

\section{Competing interests}

The authors declare that they have no competing interests.

\section{Authors' contributions}

Conceptualization, HYX; original draft preparation, HYX; review and editing, HYX, DWM, and SYL; funding acquisition, HYX, DWM, and HW. All authors read and approved the final manuscript.

\section{Author details}

'School of Mathematics and Computer Science, Shangrao Normal University, Shangrao, Jiangxi 334001, P.R. China. ${ }^{2}$ School of Mathematics and Statistics, Xidian University, Xi'an, Shaanxi 710126, P.R. China. ${ }^{3}$ Department of Informatics and Engineering, Jingdezhen Ceramic Institute, Jingdezhen, Jiangxi 333403, P.R. China.

\section{Publisher's Note}

Springer Nature remains neutral with regard to jurisdictional claims in published maps and institutional affiliations.

\section{Received: 28 September 2020 Accepted: 22 December 2020 Published online: 18 January 2021}

\section{References}

1. Xu, L., Cao, T.B.: Solutions of complex Fermat-type partial difference and differential-difference equations. Mediterr. J. Math. 15, 1-14 (2018)

2. Xu, L., Cao, T.B.: Correction to: solutions of complex Fermat-type partial difference and differential-difference equations. Mediterr. J. Math. 17, 1-4 (2020)

3. Liu, K.: Meromorphic functions sharing a set with applications to difference equations. J. Math. Anal. Appl. 359, 384-393 (2009)

4. Liu, K., Cao, T.B.: Entire solutions of Fermat type difference differential equations. Electron. J. Differ. Equ. 2013, 59 (2013)

5. Liu, K., Cao, T.B., Cao, H.Z.: Entire solutions of Fermat type differential-difference equations. Arch. Math. 99, 147-155 (2012)

6. Gross, F.: On the equation $f^{n}+g^{n}=1$. Bull. Am. Math. Soc. $72,86-88$ (1966)

7. Montel, P.: Leçons sur les Familles Normales de Fonctions Analytiques et Leurs Applications. Gauthier-Villars, Paris (1927)

8. Han, Q., Lü, F:: On the equation $f^{n}(z)+g^{n}(z)=e^{\alpha z+\beta}$. J. Contemp. Math. Anal. 54, 98-102 (2019)

9. Lü, F., Lü, W.R., Li, C.P., Xu, J.F.: Growth and uniqueness related to complex differential and difference equations. Results Math. 74, 30 (2019)

10. Liu, K., Song, C.J.: Meromorphic solutions of complex differential-difference equations. Results Math. 72, 1759-1771 (2017)

11. Naftalevich, A.: On a differential-difference equation. Mich. Math. J. 19, 59-65 (1966)

12. Naftalevich, A., Gylys, A.: On meromorphic solutions of a linear differential-difference equation with constant coefficients. Mich. Math. J. 27, 195-213 (1980)

13. Qi, X.G., Liu, Y., Yang, L.Z.: A note on solutions of some differential-difference equations. J. Contemp. Math. Anal. (Armen. Acad. Sci.) 52, 128-133 (2017)

14. Qi, X.G., Yang, L.Z.: Entire solutions of some differential-difference equations. Bull. Iran. Math. Soc. 52, 1-12 (2020)

15. Zhang, J.: On some special difference equations of Malmquist type. Bull. Korean Math. Soc. 55, 51-61 (2018)

16. Chiang, Y.M., Feng, S.J.: On the Nevanlinna characteristic of $f(z+\eta)$ and difference equations in the complex plane. Ramanujan J. 16, 105-129 (2008)

17. Halburd, R.G., Korhonen, R.J.: Difference analogue of the lemma on the logarithmic derivative with applications to difference equations. J. Math. Anal. Appl. 314, 477-487 (2006)

18. Halburd, R.G., Korhonen, R.J.: Finite-order meromorphic solutions and the discrete Painlevé equations. Proc. Lond. Math. Soc. 94, 443-474 (2007)

19. Halburd, R.G., Korhonen, R.J.: Nevanlinna theory for the difference operator. Ann. Acad. Sci. Fenn., Math. 31, 463-478 (2006)

20. Liu, M.L., Gao, L.Y.: Transcendental solutions of systems of complex differential-difference equations. Sci. Sin., Math. 49, 1-22 (2019) (in Chinese)

21. Hu, P.C., Li, B.Q.: On meromorphic solutions of nonlinear partial differential equations of first order. J. Math. Anal. Appl. $377,881-888(2011)$

22. Khavinson, D: A note on entire solutions of the eiconal equation. Am. Math Mon 102, 159-161 (1995)

23. Li, B.Q.: On entire solutions of Fermat type partial differential equations. Int. J. Math. 15, 473-485 (2004)

24. Lü, F., Li, Z.: Meromorphic solutions of Fermat type partial differential equations. J. Math. Anal. Appl. 478, 864-873 (2019) 
25. Saleeby, E.G.: Entire and meromorphic solutions of Fermat type partial differential equations. Analysis 19, 369-376 (1999)

26. Courant, R., Hilbert, D.: Methods of Mathematical Physics, Vol. II, Partial Differential Equations. Interscience, New York (1962)

27. Garabedian, P.R.: Partial Differential Equations. Wiley, New York (1964)

28. Li, B.Q.: Entire solutions of $\left(u_{z_{1}}\right)^{m}+\left(u_{z 2}\right)^{n}=e^{9}$. Nagoya Math. J. 178, 151-162 (2005)

29. Cao, T.B., Xu, L.: Logarithmic difference lemma in several complex variables and partial difference equations. Ann. Mat. 199, 767-794 (2020)

30. Cao, T.B., Korhonen, R.J.: A new version of the second main theorem for meromorphic mappings intersecting hyperplanes in several complex variables. J. Math. Anal. Appl. 444, 1114-1132 (2016)

31. Korhonen, R.J.: A difference Picard theorem for meromorphic functions of several variables. Comput. Methods Funct. Theory 12, 343-361 (2012)

32. Ronkin, L.I.: Introduction to the Theory of Entire Functions of Several Variables. Nauka, Moscow (1971) (Russian)

33. Stoll, W: Holomorphic Functions of Finite Order in Several Complex Variables. Am. Math. Soc., Providence (1974)

34. Pólya, G.: On an integral function of an integral function. J. Lond. Math. Soc. 1, 12-15 (1926)

35. Hu, P.C., Li, P., Yang, C.C.: Unicity of Meromorphic Mappings, Advances in Complex Analysis and Its Applications, vol. 1. Kluwer Academic, Dordrecht (2003)

\section{Submit your manuscript to a SpringerOpen ${ }^{\circ}$ journal and benefit from:}

- Convenient online submission

- Rigorous peer review

- Open access: articles freely available online

- High visibility within the field

- Retaining the copyright to your article

Submit your next manuscript at $\boldsymbol{\nabla}$ springeropen.com 